\title{
ATLAS Upgrades Towards the High Luminosity LHC
}

\section{Hongbo ZHU* on behalf of the ATLAS collaboration}

Deutsches Elektronen-Synchrotron DESY, Platanenallee 6, 15738 Zeuthen, Germany

E-mail: hongbo.zhu@desy.de

With the LHC having successfully collected data at center-of-mass energies of $\sqrt{s}=7 / 8 \mathrm{TeV}$, plans are actively advancing for a series of upgrades leading eventually to about five times the LHC design luminosity some ten years from now in the High-Luminosity LHC (HL-LHC) project. Coping with the high luminosity and the corresponding very high particle density will require significant changes to the ATLAS detector. Designs are developing rapidly for a replacement of the inner tracker, significant changes in the calorimeter and muon systems, and improved triggers. This article summarizes the environment expected at the HL-LHC and the status of various improvements to the ATLAS detector.

LHC on the March

20-22 November 2012

Institute for High Energy Physics, Protvino,Moscow region, Russia

${ }^{*}$ Speaker. 


\section{Introduction}

The ATLAS detector [1] is a general-purpose apparatus designed to exploit the full physics potential of the CERN Large Hadron Collider (LHC) [2], which provides proton-proton ( $p p$ ) collisions with center-of-mass energies up to $\sqrt{s}=14 \mathrm{TeV}$ at a maximum instantaneous luminosity of $\mathscr{L}=10^{34} \mathrm{~cm}^{-2} \mathrm{~s}^{-1}$. The ATLAS detector consists of three basic subsystems: the Inner Detector (ID) including Pixel, SemiConductor Tracker (SCT) and Transition Radiation Tracker (TRT), inside a $2 \mathrm{~T}$ solenoid magnetic field, the calorimetry system (Liquid Argon and Tile) and finally the Muon Spectrometer (MS) in toroidal magnetic fields of $0.5 \mathrm{~T}$. Before the first LHC long shutdown in early 2013, ATLAS recorded an integrated luminosity of $5.25 \mathrm{fb}^{-1}$ of $p p$ collisions at $\sqrt{s}=7 \mathrm{TeV}$ and $21.7 \mathrm{fb}^{-1}$ at $\sqrt{s}=8 \mathrm{TeV}$, with an overall data-taking efficiency of about $94 \%$.

In the next few years, the LHC will undergo a series of upgrades towards the High-Luminosity LHC (HL-LHC), leading ultimately to an instantaneous luminosity of $5 \times 10^{34} \mathrm{~cm}^{-2} \mathrm{~s}^{-1}$. The expected total integrated luminosity of about $3000 \mathrm{fb}^{-1}$ after ten years of HL-LHC operation will allow for studies of the properties of the new Higgs-like particle with high precision and will extend the energy scales that can be studied in high energy boson-boson scattering, to study electroweak symmetry breaking mechanism and to probe for signatures of new physics predicted by models such as SUSY and extra dimensions into the multi-TeV region $[3,4]$. The high luminosity will also impose a great challenge to ATLAS and will require significant optimizations, changes and improvements to the ATLAS detector, the data reconstruction and the analysis methods.

\section{The LHC Upgrade}

In the first LHC long shutdown in 2013-2014 (LS1), consolidation of the superconducting circuits of the LHC machine will be performed. This will allow the LHC to continue its operation (Phase-0) at the center-of-mass energy of 13-14 TeV, with an instantaneous luminosity of around $1 \times 10^{34} \mathrm{~cm}^{-2} \mathrm{~s}^{-1}$ from 2015 , and it will deliver an integrated luminosity of 50-100 $\mathrm{fb}^{-1}$. In the second long shutdown in 2018 (LS2), the LHC will upgrade its injectors and collimators to allow the instantaneous luminosity to reach $2.2 \times 10^{34} \mathrm{~cm}^{-2} \mathrm{~s}^{-1}$. The LHC will resume datataking (Phase-I) in 2019 and deliver an integrated luminosity of $300 \mathrm{fb}^{-1}$ by 2021 . In the third long shutdown in 2022 (LS3) in preparation for the HL-LHC, the LHC will install IR magnets and crab cavities. The HL-LHC will be running (Phase-II) with a leveled luminosity of $5 \times 10^{34} \mathrm{~cm}^{-2} \mathrm{~s}^{-1}$ and will deliver an integrated luminosity of $2500 \mathrm{fb}^{-1}$ over ten years, leading to a total LHC/HLLHC luminosity of $3000 \mathrm{fb}^{-1}$.

\section{The ATLAS Upgrade}

The harsher radiation environment and significantly higher detector occupancies at the HLLHC imply significant changes to most of the ATLAS systems. The major detector upgrades in the three defined phases are described in the following sections.

\subsection{ATLAS Upgrade: Phase-0}

In this two-year period (2013-2014), ATLAS has scheduled for detector consolidation works, which include the completion of the MS, installation of a new tracker evaporative cooling plant and 
detector maintenance. The central and largest ATLAS upgrade activity in Phase- 0 is the installation of the Insertable B-Layer (IBL) [5] in the present Pixel detector.

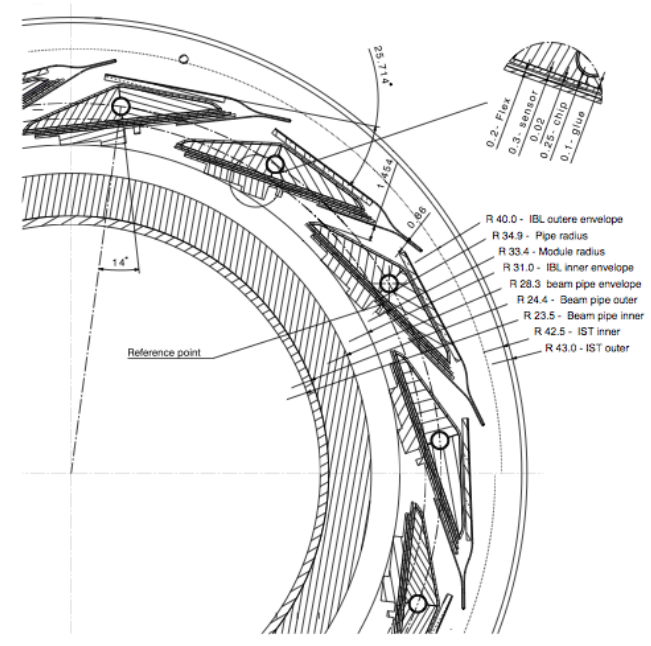

Figure 1: The IBL layers, from [6]. The inner position tube (IPT) at $R=29.3 \mathrm{~mm}$ is not shown.

The IBL is an additional $4^{\text {th }}$ pixel layer, which will be inserted between the innermost pixel layer (the B-Layer) and the beam pipe, as shown in Figure 1. To make the installation possible, a new beam pipe with reduced radius (from $r=29 \mathrm{~mm}$ to $r=25 \mathrm{~mm}$ ), made of Beryllium, is envisaged. It is expected that the IBL will improve track and vertex reconstruction and the $b$ tagging performance, which are critical to the full realization of the physics capabilities of the ATLAS experiment. The IBL consists of 14 staves, mounted directly on the beam pipe with a tilt angle of $14^{\circ}$. The center of the IBL will use 12 double-chip modules based on planar n-in-n silicon sensors, while the remaining will be equipped with 8 modules of 3D silicon sensors which are delivered as single chip sized tiles. The corresponding front-end readout chip FE-I4 [6], has been specially developed to function at high data transfer rates $(\sim 160 \mathrm{Mb} / \mathrm{s})$. The FE-I4 allows the increase of the IBL segmentation by decreasing the pixel size from the current $50 \mu \mathrm{m} \times 400 \mu \mathrm{m}$ to $50 \mu \mathrm{m} \times 250 \mu \mathrm{m}$.

\subsection{ATLAS Upgrade: Phase-I}

In this one-year period around 2018, ATLAS intends to install the new Muon Small Wheels (nMSW) and finer granularity Level-1 readout for the LAr calorimeter, upgrade the very forward taggers (AFP) and introduce new trigger schemes (including the Fast Tracker Trigger) to handle luminosities well beyond the nominal value and therefore improve the physics performance of the experiment [7].

The Muon Small Wheels (MSW), shown in Figure 2, are located in between the end-cap calorimeter and the end-cap toroid $(|\eta|=1.3-2.7)$, which is the region of highest cavern background in the MS. In the Phase-I upgrade, the nMSW should be designed and built to replace the existing ones. The nMSW must ensure efficient track reconstruction at high particle rate (at the high luminosity of $\mathscr{L}=5 \times 10^{34} \mathrm{~cm}^{-2} \mathrm{~s}^{-1}$ ) and position resolution better than $100 \mu \mathrm{m}$. Furthermore, the nMSW will be integrated into the Level-1 trigger. Several detector technologies are 

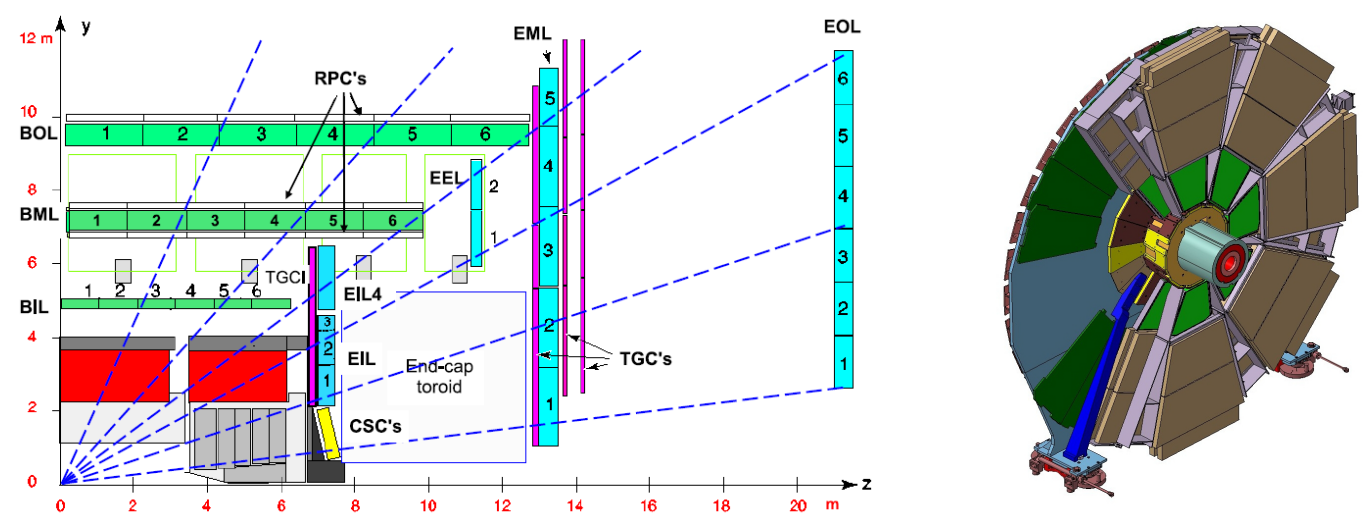

Figure 2: Left: A $z-y$ view of $1 / 4$ of the ATLAS detector. The blue boxes indicate the end-cap MDT chambers and the yellow box CSC. Right: A view of a small wheel mounted on the disk shielding, from [7].

under investigation: small diameter Muon Drift Tubes (sMDT) complemented with faster trigger chambers - Resistive Plate Chambers (RPC) or Thin Gap Chambers (TGC), and Micromegas.

Also, in the Phase-I upgrade more sophisticated trigger schemes will be implemented. The Fast Tracker Trigger (FTK) is a highly parallel hardware system designed to operate at the Level-1 trigger output rate and provide global tracks reconstructed in the ID with resolution comparable to the full offline reconstruction as input of the Level-2 trigger processing. It utilizes associative memories for pattern recognition and fast FPGAs for track reconstruction. The FTK is expected to dramatically improve the performance of track based isolation and $b$-tagging with nearly no dependence on pile-up interactions. In addition, there are proposals on combination of trigger objects at Level-1 (topological triggers) and the implementation of full granularity readout of the calorimeter. The latter will strongly improve the triggering capabilities for electrons and photons at Level-1.

\subsection{ATLAS Upgrade: Phase-II}

The Phase-II upgrade for the HL-LHC around 2022 aims to increase the instantaneous luminosity to $5 \times 10^{34} \mathrm{~cm}^{-2} \mathrm{~s}^{-1}$, presenting significant challenges to the operation and performance of the ATLAS detector systems. This requires the full replacement of the ID and further trigger/calorimeter upgrades [4].

The increased luminosity and the accumulated radiation damage will render the current ID no longer suitable for long term operation. A replacement with a new all-silicon tracker is planned to maintain the tracking performance in high occupancy environment and to cope with the ten times higher radiation level. New technologies are used to ensure higher detector granularity, lower material budget and increased radiation hardness. The current baseline design of the new tracker is depicted in Figure 3. It consists of 4 Pixel and 5 silicon strip layers (3 short-strip layers +2 long-strip layers) in the central region, and 6 Pixel disks followed by 7 silicon strip double-sided disks on each side of the end-caps. The tracker covers up to $|\eta|=2.5$ (Pixel up to $|\eta|=2.7$ ) and aims to provide at least 14 silicon hits for each reconstructed track.

The HL-LHC conditions will also have a significant impact on the LAr calorimeters. The front-end electronics have to be replaced, due to both radiation damage and the need for ATLAS to 


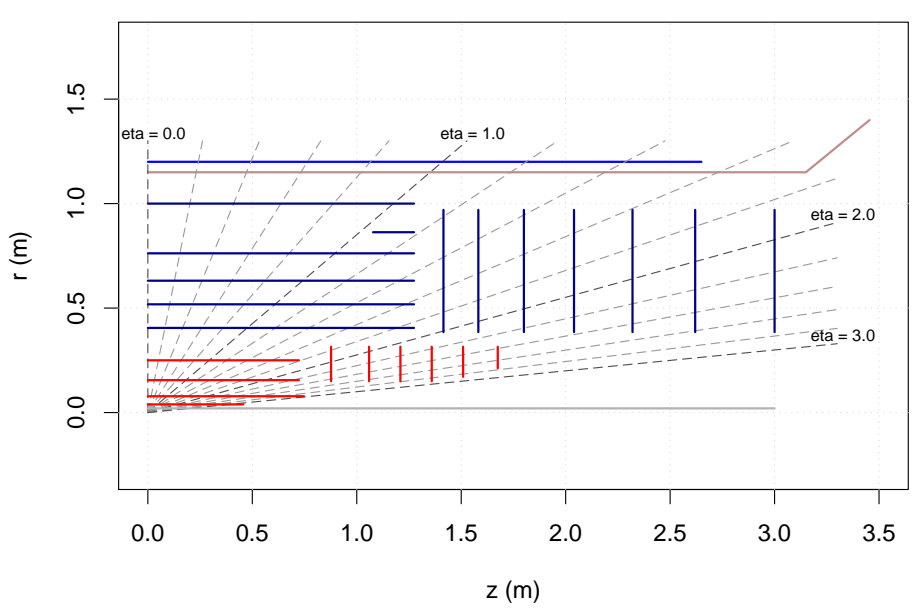

Figure 3: The baseline layout of the replacement tracker, from [4].

upgrade the trigger system, with the latter requiring real-time performance capabilities that the current electronics cannot satisfy. Furthermore, the performance of the Forward Calorimeter (FCal) could be compromised. To maintain the FCal functionalities under the HL-LHC conditions, two possible solutions are under evaluation: complete replacement of the FCal, or the alternative installation of a small calorimeter in front of the current FCal (called "Mini-FCal"). The Mini-FCal would reduce the ionization and heat loads of the FCal to acceptable levels.

\section{Summary}

ATLAS has fully planned the upgrade of the apparatus in three phases towards the high luminosity LHC. With all the improvements, ATLAS is expected to preserve and even improve its detector performance under the high luminosity conditions and can fully exploit the physics potential of the LHC/HL-LHC.

\section{References}

[1] ATLAS Collaboration, The ATLAS Experiment at the CERN Large Hadron Collider, JINST 3 (2008) S08003.

[2] L. Evans and P. Bryant, LHC Machine, JINST 3 (2008) S08001.

[3] ATLAS Collaboration, Physics at a High-Luminosity LHC with ATLAS, ATL-PHYS-PUB-2012-004.

[4] ATLAS Collaboration, Letter of Intent for the Phase-II Upgrade of the ATLAS Experiment, CERN-LHCC-2012-022; LHCC-I-023.

[5] ATLAS Collaboration, ATLAS Insertable B-Layer Technical Design Report, CERN-LHCC-2010-013; ATLAS-TDR-19; ATLAS Insertable B-Layer Technical Design Report Addendum, CERN-LHCC-2012-009; ATLAS-TDR-19-ADD-1.

[6] The ATLAS IBL Collaboration, Prototype ATLAS IBL modules using the FE-I4A front-end readout chip, JINST 7 (2012) P11010.

[7] ATLAS Collaboration, Letter of Intent for the Phase-I Upgrade of the ATLAS Experiment, CERN-LHCC-2011-012; LHCC-I-20. 